PROCEEDINGS OF THE

AMERICAN MATHEMATICAL SOCIETY

Volume 140, Number 2, February 2012, Pages 581-588

S 0002-9939(2011)11134-1

Article electronically published on July 20, 2011

\title{
NONDEGENERACY OF ENTIRE SOLUTIONS OF A SINGULAR LIOUVILLLE EQUATION
}

\author{
MANUEL DEL PINO, PIERPAOLO ESPOSITO, AND MONICA MUSSO
}

(Communicated by Matthew J. Gursky)

\begin{abstract}
We establish nondegeneracy of the explicit family of finite mass solutions of the Liouvillle equation with a singular source of integer multiplicity, in the sense that all bounded elements in the kernel of the linearization correspond to variations along the parameters of the family.
\end{abstract}

\section{INTRODUCTION AND STATEMENT OF THE MAIN RESULT}

Liouville type equations with singular sources in two space dimensions arise in various interesting contexts and have been the object of many studies in recent years. The model problem of this type is the equation

$$
\Delta u+e^{u}=4 \pi N \delta_{0} \quad \text { in } \mathbb{R}^{2}
$$

where $\delta_{0}$ designates the Dirac mass at the origin and $N$ is a nonnegative integer.

Singular Liouville equations appear for instance in the Abelian Maxwell-Higgs and Chern-Simons-Higgs theories of superconductivity, in the self-dual regime. In the latter model, a mean field form of problem (1) on the torus becomes the limiting equation for non-topological condensates as shown in [13, 15]. The number $N$ represents vortex multiplicity in that context, so that the most interesting case is precisely that in which it is a positive integer. This type of singular equation arises also in Euler flows [2, 17] and naturally in the construction of singular conformal metrics in $\mathbb{R}^{2}$. We refer the reader to [11, 12, 16] and references therein for recent developments in this subject and related issues.

In [14, with the aid of Liouville's formula (see (3) below), it is shown that all solutions of this problem with finite mass $\int_{\mathbb{R}^{2}} e^{u}<+\infty$ are given, in complex notation, by the family

$$
U_{\mu, a}(z)=\log \frac{8 \mu^{2}(N+1)^{2}|z|^{2 N}}{\left(\mu^{2}+\left|z^{N+1}-a\right|^{2}\right)^{2}}, \quad \mu \in \mathbb{R}, \quad a \in \mathbb{C},
$$

for which we observe

$$
\int_{\mathbb{R}^{2}} e^{u}=8 \pi(N+1)
$$

Received by the editors November 23, 2010.

2010 Mathematics Subject Classification. Primary 35J25, 35B40, 35J10.

Key words and phrases. Singular Liouville equation, linearization, nondegeneracy.

This research has been partly supported by grants FIRB-IDEAS (2008), project "Geometrical aspects in PDEs", Fondecyt Grants 1070389, 1080099, Fondo Basal CMM and CAPDE-Anillo ACT-125.

(C)2011 American Mathematical Society 
Our purpose in this paper is to prove the $L^{\infty}$-nondegeneracy of these solutions.

The linearized operator for (1) at $U_{\mu, a}$ is given by

$$
L(\phi):=\Delta \phi+e^{U_{\mu, a}(z)} \phi
$$

or

Since

$$
L(\phi)=\Delta \phi+\frac{8 \mu^{2}(N+1)^{2}|z|^{2 N}}{\left(\mu^{2}+\left|z^{N+1}-a\right|^{2}\right)^{2}} \phi .
$$

it follows that the functions

$$
\Delta U_{\mu, a}(z)+e^{U_{\mu, a}(z)}=4 \pi \delta_{0}
$$

$$
Z_{1}:=\partial_{\mu} U_{\mu, a}, \quad Z_{2}:=\partial_{a_{1}} U_{\mu, a}, \quad Z_{3}:=\partial_{a_{2}} U_{\mu, a} \quad\left(a=a_{1}+i a_{2}\right)
$$

are bounded solutions of the equation $L(Z)=0$. Explicitly we have

$$
\begin{aligned}
& Z_{1}=-\frac{2}{\mu} \frac{\mu^{2}-\left|z^{N+1}-a\right|^{2}}{\mu^{2}+\left|z^{N+1}-a\right|^{2}}, \quad Z_{2}=2 \frac{\operatorname{Re}\left(z^{N+1}-a\right)}{\mu^{2}+\left|z^{N+1}-a\right|^{2}}, \\
& Z_{3}=-2 \frac{\operatorname{Im}\left(z^{N+1}-a\right)}{\mu^{2}+\left|z^{N+1}-a\right|^{2}} .
\end{aligned}
$$

We prove the $L^{\infty}$-nondegeneracy of the solutions $U_{\mu, a}$ in the following sense:

Theorem 1. If $\phi \in L^{\infty}\left(\mathbb{R}^{2}\right)$ solves the equation $L(\phi)=0$, then $\phi$ must be a linear combination of the functions $Z_{1}, Z_{2}, Z_{3}$.

Nondegeneracy is an important ingredient in the construction of solutions to problems involving small parameters and concentration phenomena in which, after suitable blowing-up around a concentration point, one sees a limiting equation. Theorem 1 is known for $N=0$; see [1. This property has been used for instance in [1, 6, 9] to build solutions with multiple concentration points for the problem

$$
\Delta u+\varepsilon^{2} e^{u}=0 \quad \text { in } \Omega, \quad u=0 \quad \text { on } \partial \Omega,
$$

as $\varepsilon \rightarrow 0$ where $\Omega$ is a bounded domain in $\mathbb{R}^{2}$. See also [3, 4, 7, 10] for related constructions. See also [8] for the case in which a singular source $4 \pi N \delta_{P}$ is present in the right hand side of the equation and $N$ is not an integer.

In the case that $N$ is an integer, in [5] we have built concentrating solutions at a single point in the simply connected case with the aid of Liouville's representation formula. Theorem 1 may represent a major step in the construction of new solutions for this and related problems with exponential nonlinearities.

As we have mentioned above, in [1] Theorem 1 was proven for $N=0$. We observe that in that case the simple transformation

$$
\tilde{\phi}(z):=\phi(a+\mu z)
$$

reduces the equation $L(\phi)=0$ to

$$
\Delta \tilde{\phi}+\frac{8}{\left(1+|z|^{2}\right)^{2}} \tilde{\phi}=0 .
$$

Then, using usual polar coordinates $z=\rho e^{i \theta}$ and decomposing $\tilde{\phi}$ into a Fourier series

$$
\tilde{\phi}(\rho, \theta)=\sum_{k \geq 0} a_{k}(\rho) \cos (k \theta)+b_{k}(\rho) \sin (k \theta),
$$

we find that the equation decouples into independent ODEs for each of the coefficients $a_{k}, b_{k}$. These equations can be solved one by one, then leading easily to 
the desired result. When $N>0$, the parameter $\mu$ can be absorbed similarly, but we cannot do so with $a$. Then, we have to live with the fact that the potential in the equation is nonradial, which makes impossible the decoupling. We are able to circumvent this nontrivial difficulty by an expansion of the solution into a suitable orthonormal system, different from the trigonometric one, suitably adapted to the equation. We devote the rest of the paper to carrying out the proof.

\section{The proof of Theorem 1}

As we have commented above, by scaling we can eliminate the parameter $\mu$ in the equation so that we have to prove that if $\phi \in L^{\infty}\left(\mathbb{R}^{2}\right)$ solves the equation

$$
L(\phi)=\Delta \phi+\frac{8(N+1)^{2}|z|^{2 N}}{\left(1+\left|z^{N+1}-a\right|^{2}\right)^{2}} \phi=0 \text { in } \mathbb{R}^{2},
$$

then $\phi$ is a linear combination of the functions $Z_{1}, Z_{2}, Z_{3}$ in (2) for $\mu=1$.

Let us recall the Liouville formula: given a holomorphic function $f$ on $\mathbb{C}$, the function

$$
U(z)=\ln \frac{8\left|f^{\prime}(z)\right|^{2}}{\left(1+|f(z)|^{2}\right)^{2}}
$$

solves the equation

$$
\Delta U+e^{U}=0
$$

in the set

$$
\left\{z \in \mathbb{C} / f^{\prime}(z) \neq 0\right\} \text {. }
$$

If $f^{\prime}$ now has a zero at the origin of multiplicity $N$, the function

$$
\ln \frac{8\left|f^{\prime}(z)\right|^{2}}{\left(1+|f(z)|^{2}\right)^{2}}-\ln |z|^{2 N}
$$

solves the equation $\Delta U+|z|^{2 N} e^{U}=0$ in the set $\left\{z \in \mathbb{C} \backslash\{0\} / f^{\prime}(z) \neq 0\right\}$. The choice

$$
f(z)=z^{N+1}\left(1+\tau z^{k}\right)-a, \quad k \geq 0,
$$

leads to a family

$$
U_{\tau, k}(z)=\ln \frac{8(N+1)^{2}\left|1+\tau \frac{N+1+k}{N+1} z^{k}\right|^{2}}{\left(1+\left|z^{N+1}\left(1+\tau z^{k}\right)-a\right|^{2}\right)^{2}}, \quad \tau \in \mathbb{C},
$$

of solutions of

$$
\Delta U+|z|^{2 N} e^{U}=0
$$

in

$$
\mathbb{C} \backslash\left\{z \in \mathbb{C}: 1+\tau \frac{N+1+k}{N+1} z^{k}=0\right\} .
$$

The derivative of $U_{\tau, k}$ in $\tau$ at $\tau=0$

$$
\phi_{k}:=\left.\frac{N+1}{N+1+k} \partial_{\tau} U_{\tau, k}\right|_{\tau=0}=z^{k}\left(1-2 \frac{N+1}{N+1+k} \frac{z^{N+1} \overline{z^{N+1}-a}}{1+\left|z^{N+1}-a\right|^{2}}\right)
$$

solves $L\left(\phi_{k}\right)=0$ in $\mathbb{C}$, and in particular, $\phi_{0}^{1}=\operatorname{Re} \phi_{0}$ and $\phi_{k}^{1}=\operatorname{Re} \phi_{k}, \phi_{k}^{2}=\operatorname{Im} \phi_{k}$ are real solutions for $k \geq 1$. We claim that 
Claim 1. Every bounded solution $\phi$ of $L(\phi)=0$ is a linear combination of $\phi_{0}^{1}$ and $\phi_{k}^{i}, k \geq 1$ and $i=1,2$ :

$$
\phi=a_{0} \phi_{0}^{1}+\sum_{k \geq 1}\left(a_{k} \phi_{k}^{1}+b_{k} \phi_{k}^{2}\right)
$$

Proof of Claim 1. The key idea is that, for $\rho$ small, the functions $\phi_{0}^{1}\left(\rho e^{i \theta}\right)$, $\frac{1}{\rho^{k}} \phi_{k}\left(\rho e^{i \theta}\right)$ and $\frac{1}{\rho^{k}} \overline{\phi_{k}}\left(\rho e^{i \theta}\right)$ with $k \geq 1$ are very close to the complex Fourier basis $\frac{1}{2 \pi} e^{i k \theta}, k \in \mathbb{Z}$, and then form a complete set in $L^{2}\left(\partial B_{\rho}(0), \mathbb{C}\right)$.

By an integration by parts first we can compute for $k \neq 0$

$$
\frac{1}{2 \pi} \int_{S^{1}} \phi_{0}^{1}\left(\rho e^{i \theta}\right) d \theta=1+O\left(\rho^{N+1}\right), \quad \frac{1}{2 \pi} \int_{S^{1}} e^{i k \theta} \phi_{0}^{1}\left(\rho e^{i \theta}\right) d \theta=O\left(\frac{\rho^{N+1}}{|k|}\right)
$$

as $\rho \rightarrow 0^{+}$(uniformly in $k$ ). A more careful integration by parts yields that for $j \geq 1$ :

$$
\begin{aligned}
& \frac{1}{2 \pi} \int_{S^{1}} e^{i k \theta} \frac{1}{\rho^{j}} \phi_{j}\left(\rho e^{i \theta}\right) d \theta \\
& =\frac{1}{2 \pi} \int_{S^{1}} e^{i(k+j) \theta}\left(1-2 \frac{N+1}{N+1+j} \frac{\rho^{N+1} e^{i(N+1) \theta} \overline{\rho^{N+1} e^{i(N+1) \theta}-a}}{1+\left|\rho^{N+1} e^{i(N+1) \theta}-a\right|^{2}}\right) d \theta \\
& \left.=\delta_{k=-j}-\frac{N+1}{\pi(N+1+j)} \rho^{N+1} \int_{S^{1}} e^{i(k+j+N+1) \theta} \frac{\frac{\rho^{N+1} e^{i(N+1) \theta}-a}{1+\left|\rho^{N+1} e^{i(N+1) \theta}-a\right|^{2}}}{\rho^{N+1}}\right)-\delta_{k \neq-j-N-1} \frac{i(N+1) \rho^{N+1}}{\pi(N+1+j)(k+j+N+1)} \\
& =\delta_{k=-j}+\delta_{k=-j-N-1} O\left(\frac{1+j}{N+1+j}\right) \\
& \quad \times \int_{S^{1}} e^{i(k+j+N+1) \theta} \partial_{\theta}\left[\frac{\rho^{N+1} e^{i(N+1) \theta}-a}{1+\left|\rho^{N+1} e^{i(N+1) \theta}-a\right|^{2}}\right] d \theta \\
& =\delta_{k=-j}+\delta_{k=-j-N-1} O\left(\frac{\rho^{N+1}}{N+1+j}\right) \\
& \quad+\delta_{k \neq-j-N-1} O\left(\frac{\rho^{N+1}}{(N+1+j)|k+j+N+1|}\right)
\end{aligned}
$$

as $\rho \rightarrow 0^{+}$(uniformly in $k$ ). Similarly, for $j \geq 1$ as $\rho \rightarrow 0^{+}$(uniformly in $k$ ) we have that

$$
\begin{aligned}
& \frac{1}{2 \pi} \int_{S^{1}} e^{i k \theta} \frac{1}{\rho^{j}} \overline{\phi_{j}}\left(\rho e^{i \theta}\right) d \theta \\
& =\frac{1}{2 \pi} \int_{S^{1}} e^{i(k-j) \theta}\left(1-2 \frac{N+1}{N+1+j} \frac{\rho^{N+1} e^{-i(N+1) \theta}\left[\rho^{N+1} e^{i(N+1) \theta}-a\right]}{1+\left|\rho^{N+1} e^{i(N+1) \theta}-a\right|^{2}}\right) d \theta \\
& =\delta_{k=j}+\delta_{k=j+N+1} O\left(\frac{\rho^{N+1}}{N+1+j}\right)+\delta_{k \neq j+N+1} O\left(\frac{\rho^{N+1}}{(N+1+j)|k-j-N-1|}\right) .
\end{aligned}
$$

Letting $\psi \in L^{2}\left(\partial B_{\rho}(0), \mathbb{C}\right)$ in the form $\psi\left(\rho e^{i \theta}\right)=\sum_{k \in \mathbb{Z}} c_{k} e^{i k \theta}$, we can compute

$$
\tilde{c}_{0}:=\frac{1}{2 \pi} \int_{S^{1}} \psi\left(\rho e^{i \theta}\right) \phi_{0}^{1}\left(\rho e^{i \theta}\right) d \theta=c_{0}+\rho^{N+1} O\left(\sum_{k \in \mathbb{Z}} \frac{\left|c_{k}\right|}{|k|+1}\right)
$$


and for $j \geq 1$

$$
\begin{aligned}
\tilde{c}_{j} & :=\frac{1}{2 \pi} \int_{S^{1}} \psi\left(\rho e^{i \theta}\right) \frac{1}{\rho^{j}} \overline{\phi_{j}}\left(\rho e^{i \theta}\right) d \theta \\
& =c_{j}+\frac{\rho^{N+1}}{N+1+j} O\left(\sum_{k \in \mathbb{Z}} \frac{\left|c_{k}\right|}{|k-j-N-1|+1}\right), \\
\tilde{c}_{-j} & :=\frac{1}{2 \pi} \int_{S^{1}} \psi\left(\rho e^{i \theta}\right) \frac{1}{\rho^{j}} \phi_{j}\left(\rho e^{i \theta}\right) d \theta \\
& =c_{-j}+\frac{\rho^{N+1}}{N+1+j} O\left(\sum_{k \in \mathbb{Z}} \frac{\left|c_{k}\right|}{|k+j+N+1|+1}\right) .
\end{aligned}
$$

We consider the operator

$$
T: c=\left(\ldots, c_{-1}, c_{0}, c_{1}, \ldots\right) \in l^{2} \rightarrow \tilde{c}=\left(\ldots, \tilde{c}_{-1}, \tilde{c}_{0}, \tilde{c}_{1}, \ldots\right) \in l^{2} .
$$

In view of

$$
\sum_{k \in \mathbb{Z}} \frac{\left|c_{k}\right|}{\left|k+j_{0}\right|+1} \leq\left(\sum_{k \in \mathbb{Z}}\left|c_{k}\right|^{2}\right)^{\frac{1}{2}}\left(\sum_{k \in \mathbb{Z}} \frac{1}{\left(\left|k+j_{0}\right|+1\right)^{2}}\right)^{\frac{1}{2}}=\|c\|_{l^{2}}\left(\sum_{k \in \mathbb{Z}} \frac{1}{(|k|+1)^{2}}\right)^{\frac{1}{2}}
$$

for every given $j_{0}$, we have shown so far that

$$
\left|\tilde{c}_{0}-c_{0}\right|=O\left(\rho^{N+1}\|c\|_{l^{2}}\right), \quad\left|\tilde{c}_{j}-c_{j}\right|+\left|\tilde{c}_{-j}-c_{-j}\right|=O\left(\frac{\rho^{N+1}}{j+1}\|c\|_{l^{2}}\right),
$$

and then

$$
\|T-\mathrm{Id}\| \leq C^{\prime} \rho^{N+1}\left(\sum_{j \geq 0} \frac{1}{(j+1)^{2}}\right)^{\frac{1}{2}} \leq C \rho^{N+1} .
$$

In conclusion, for $\rho$ small we have that $T$ is an invertible operator. If $\psi \in$ $L^{2}\left(\partial B_{\rho}(0), \mathbb{R}\right)$ is such that

$$
\int_{S^{1}} \psi\left(\rho e^{i \theta}\right) \phi_{0}^{1}\left(\rho e^{i \theta}\right) d \theta=\int_{S^{1}} \psi\left(\rho e^{i \theta}\right) \phi_{k}^{j}\left(\rho e^{i \theta}\right) d \theta=0 \quad \forall k \geq 1, j=1,2,
$$

then $\tilde{c}=0$, and by injectivity of $T$ we deduce that the Fourier coefficients $c_{j}$ of $\psi\left(\rho e^{i \theta}\right)$ vanish yielding to $\psi=0$. This means that, for $\rho$ small, the space $L^{2}\left(\partial B_{\rho}(0), \mathbb{R}\right)$ coincides with the closure in $L^{2}$-norm of

$$
\text { Span }\left\{\phi_{0}^{1}, \phi_{k}^{j}, k \geq 1, j=1,2\right\} .
$$

In particular, every bounded solution $\phi$ of $L(\phi)=0$ in $\mathbb{C}$ can be written on $\partial B_{\rho}(0)$, for $\rho$ small, as

$$
\phi\left(\rho e^{i \theta}\right)=a_{0} \phi_{0}^{1}\left(\rho e^{i \theta}\right)+\sum_{k \geq 1}\left(a_{k} \phi_{k}^{1}\left(\rho e^{i \theta}\right)+b_{k} \phi_{k}^{2}\left(\rho e^{i \theta}\right)\right),
$$

for suitable $a_{j}$ and $b_{j}$. By regularity theory $\phi \in C^{\infty}(\mathbb{C})$, and then $\left.\phi\right|_{\partial B_{\rho}(0)} \in$ $C^{\infty}\left(\partial B_{\rho}(0)\right)$. Arguing as for the Fourier coefficients, it is easily seen (with tedious computations, due to the almost orthogonality of $\phi_{k}\left(\rho e^{i \theta}\right)$ ) that $a_{k}$ and $b_{k}$ tend to zero as $k \rightarrow+\infty$ faster than any power of $k$. In particular, the function

$$
\hat{\phi}(z)=a_{0} \phi_{0}^{1}(z)+\sum_{k \geq 1}\left[a_{k} \phi_{k}^{1}(z)+b_{k} \phi_{k}^{2}(z)\right]
$$


is well defined, is in $C^{\infty}(\mathbb{C})$ and satisfies $L(\hat{\phi})=0$ in $\mathbb{C}$. Since $\phi=\hat{\phi}$ on $\partial B_{\rho}(0)$ and $\delta=\phi-\hat{\phi}$ satisfies $L(\delta)=0$ in $\mathbb{C}$, an integration by parts yields to

$$
\int_{B_{\rho}(0)}|\nabla \delta|^{2}=\int_{B_{\rho}(0)} V \delta^{2}-\int_{B_{\rho}(0)} L(\delta) \delta=\int_{B_{\rho}(0)} V \delta^{2} \leq C \rho^{2 N} \int_{B_{\rho}(0)} \delta^{2},
$$

where $V(z)=\frac{8(N+1)^{2}|z|^{2 N}}{\left(1+\left|z^{N+1}-a\right|^{2}\right)^{2}}$. As soon as $C \rho^{2 N}<\lambda_{1}\left(B_{\rho}(0)\right)\left(\lambda_{1}\right.$ being the first eigenvalue of $-\Delta$ with Dirichlet boundary conditions), we get that necessarily $\delta=0$ in $B_{\rho}(0)$. Then, for $\rho$ small we have that $\delta=0$ in $B_{\rho}(0)$, and by the strong maximum principle $\delta=0$ in $\mathbb{C}$. So we have shown that

$$
\phi(z)=a_{0} \phi_{0}^{1}(z)+\sum_{k \geq 1}\left[a_{k} \phi_{k}^{1}(z)+b_{k} \phi_{k}^{2}(z)\right]
$$

in $\mathbb{C}$.

Let us look now at the behavior of $\phi(z)$ as $|z| \rightarrow+\infty$. Since the only bounded components in $\phi(z)$ are $\phi_{0}^{1}$ and $\phi_{N+1}^{1}, \phi_{N+1}^{2}$, we claim that

Claim 2. $a_{k}=b_{k}=0$ for $k \neq 0, N+1$.

Proof of Claim 2. Also in this case we will use that the components of $\phi$ are very close to the Fourier basis as $|z| \rightarrow+\infty$. Indeed, observe that

$$
\frac{z^{N+1} \overline{z^{N+1}-a}}{1+\left|z^{N+1}-a\right|^{2}}=1+O\left(\frac{1}{|z|^{N+1}}\right) \quad \text { as }|z| \rightarrow+\infty,
$$

and then

$$
\phi_{k}(z)=z^{k}\left(\frac{k-N-1}{N+1+k}+O\left(\frac{1}{|z|^{N+1}}\right)\right)
$$

at infinity (uniformly in $k \geq 0$ ). More explicitly, we have that

$$
\begin{aligned}
& \phi_{0}^{1}(z)=-1+O\left(\frac{1}{|z|^{N+1}}\right), \quad \phi_{k}^{1}(z)=\frac{k-N-1}{N+1+k}|z|^{k} \cos (k \theta)\left(1+O\left(\frac{1}{|z|^{N+1}}\right)\right), \\
& \phi_{k}^{2}(z)=\frac{k-N-1}{N+1+k}|z|^{k} \sin (k \theta)\left(1+O\left(\frac{1}{|z|^{N+1}}\right)\right)
\end{aligned}
$$

as $|z| \rightarrow+\infty$. Using Cauchy-Schwartz's inequality, we compute

$$
\begin{aligned}
\frac{1}{R} \int_{\partial B_{R}(0)} \phi^{2}= & \pi\left(\sum_{k \geq 0} \frac{(k-N-1)^{2}}{(N+1+k)^{2}} R^{2 k} a_{k}^{2}+\sum_{k \geq 1} \frac{(k-N-1)^{2}}{(N+1+k)^{2}} R^{2 k} b_{k}^{2}\right) \\
& +o\left(\sum_{k, j}\left|\frac{k-N-1}{N+1+k} \| \frac{j-N-1}{N+1+j}\right| R^{k+j}\left(\left|a_{k}\right|\left|a_{j}\right|+\left|b_{k}\right|\left|b_{j}\right|+\left|a_{k} \| b_{j}\right|\right)\right) \\
= & \pi(1+o(1))\left(\sum_{k \geq 0} \frac{(k-N-1)^{2}}{(N+1+k)^{2}} R^{2 k} a_{k}^{2}+\sum_{k \geq 1} \frac{(k-N-1)^{2}}{(N+1+k)^{2}} R^{2 k} b_{k}^{2}\right)
\end{aligned}
$$

as $R \rightarrow+\infty$. Since $\phi$ is bounded in $\mathbb{C}$, we have that $\frac{1}{R} \int_{\partial B_{R}} \phi^{2}$ is bounded in $R$, and then

$$
\sum_{k \geq 0} \frac{(k-N-1)^{2}}{(N+1+k)^{2}} R^{2 k} a_{k}^{2}+\sum_{k \geq 1} \frac{(k-N-1)^{2}}{(N+1+k)^{2}} R^{2 k} b_{k}^{2}
$$

is bounded in $R$. Then $a_{k}=0$ and $b_{k}=0$ for $k \geq 1$ unless $k=N+1$. 
For a bounded solution $\phi$ of $L(\phi)=0$ we have then shown that

$$
\phi(z)=a_{0} \phi_{0}^{1}(z)+a_{N+1} \phi_{N+1}^{1}(z)+b_{N+1} \phi_{N+1}^{2}(z) .
$$

To establish the validity of Theorem 1, we need simply to rewrite $\phi_{0}$ and $\phi_{N+1}$ in a more explicit way. We have that

$$
\phi_{0}(z)=1-2 \frac{z^{N+1} \overline{z^{N+1}-a}}{1+\left|z^{N+1}-a\right|^{2}}=\frac{1-\left|z^{N+1}-a\right|^{2}}{1+\left|z^{N+1}-a\right|^{2}}-2 a \frac{\overline{z^{N+1}-a}}{1+\left|z^{N+1}-a\right|^{2}}
$$

and

$$
\begin{aligned}
\phi_{N+1}(z) & =z^{N+1}\left(1-\frac{z^{N+1} \overline{z^{N+1}-a}}{1+\left|z^{N+1}-a\right|^{2}}\right) \\
& =a \frac{1-\left|z^{N+1}-a\right|^{2}}{1+\left|z^{N+1}-a\right|^{2}}+\frac{z^{N+1}-a}{1+\left|z^{N+1}-a\right|^{2}}-a^{2} \frac{\overline{z^{N+1}-a}}{1+\left|z^{N+1}-a\right|^{2}} .
\end{aligned}
$$

In real form we can then write that

$$
\phi_{0}^{1}(z)=\frac{1-\left|z^{N+1}-a\right|^{2}}{1+\left|z^{N+1}-a\right|^{2}}-2 a^{1} \operatorname{Re} \frac{z^{N+1}-a}{1+\left|z^{N+1}-a\right|^{2}}-2 a^{2} \operatorname{Im} \frac{z^{N+1}-a}{1+\left|z^{N+1}-a\right|^{2}}
$$

and

$$
\begin{aligned}
\phi_{N+1}^{1}(z)=a^{1} & \frac{1-\left|z^{N+1}-a\right|^{2}}{1+\left|z^{N+1}-a\right|^{2}}+\left(1-\left(a^{1}\right)^{2}+\left(a^{2}\right)^{2}\right) \operatorname{Re} \frac{z^{N+1}-a}{1+\left|z^{N+1}-a\right|^{2}} \\
& -2 a^{1} a^{2} \operatorname{Im} \frac{z^{N+1}-a}{1+\left|z^{N+1}-a\right|^{2}}
\end{aligned}
$$

and

$$
\begin{aligned}
\phi_{N+1}^{2}(z)=a^{2} & \frac{1-\left|z^{N+1}-a\right|^{2}}{1+\left|z^{N+1}-a\right|^{2}}-2 a^{1} a^{2} \operatorname{Re} \frac{z^{N+1}-a}{1+\left|z^{N+1}-a\right|^{2}} \\
& +\left(1+\left(a^{1}\right)^{2}-\left(a^{2}\right)^{2}\right) \operatorname{Im} \frac{z^{N+1}-a}{1+\left|z^{N+1}-a\right|^{2}},
\end{aligned}
$$

where $a=a^{1}+i a^{2}$. As a conclusion, the function $\phi$ can be written as

$$
\begin{aligned}
\phi= & \left(a_{0}+a_{N+1} a^{1}+b_{N+1} a^{2}\right) \frac{1-\left|z^{N+1}-a\right|^{2}}{1+\left|z^{N+1}-a\right|^{2}} \\
& +\left[-2 a_{0} a^{1}+a_{N+1}\left(1-\left(a^{1}\right)^{2}+\left(a^{2}\right)^{2}\right)-2 b_{N+1} a^{1} a^{2}\right] \operatorname{Re} \frac{z^{N+1}-a}{1+\left|z^{N+1}-a\right|^{2}} \\
& +\left[-2 a_{0} a^{2}-2 a_{N+1} a^{1} a^{2}+b_{N+1}\left(1+\left(a^{1}\right)^{2}-\left(a^{2}\right)^{2}\right)\right] \operatorname{Im} \frac{z^{N+1}-a}{1+\left|z^{N+1}-a\right|^{2}}
\end{aligned}
$$

and Theorem 1 is established.

\section{REFERENCES}

[1] S. Baraket, F. Pacard, Construction of singular limits for a semilinear elliptic equation in dimension 2, Calc. Var. Partial Differential Equations 6 (1998), no. 1, 1-38. MR.1488492 (98j:35057)

[2] E. Caglioti, P.L. Lions, C. Marchioro, M. Pulvirenti, A special class of stationary flows for two-dimensional Euler equations: a statistical mechanics description, Part II. Comm. Math. Phys. 174 (1995), no. 2, 229-260. MR.1362165 (96k:82059)

[3] C.-C. Chen, C.-S. Lin, Topological degree for a mean field equation on Riemann surfaces. Comm. Pure Appl. Math. 56 (2003), no. 12, 1667-1727. MR2001443(2004h:35065) 
[4] J. Dávila, M. del Pino, M. Musso, J. Wei, Singular limits of a two-dimensional boundary value problem arising in corrosion modelling. Arch. Ration. Mech. Anal. 182 (2006), no. 2, 181-221. MR2259331(2007i:35091)

[5] M. del Pino, P. Esposito, M. Musso,Two-dimensional Euler flows with concentrated vorticities. Trans. Amer. Math. Soc. 362 (2010), no. 12, 6381-6395. MR.2678979

[6] M. del Pino, M. Kowalczyk, M. Musso, Singular limits in Liouville-type equations. Calc. Var. Partial Differential Equations 24 (2005), no. 1, 47-81. MR2157850(2006h:35089)

[7] M. del Pino, M. Musso, B. Ruf, New solutions for Trudinger-Moser critical equations in $\mathbb{R}^{2}$. J. Funct. Anal. 258 (2010), no. 2, 421-457. MR2557943 (2010k:35092)

[8] P. Esposito, Blow up solutions for a Liouville equation with singular data. SIAM J. Math. Anal. 36 (2005), no. 4, 1310-1345. MR2139452 (2005m:35088)

[9] P. Esposito, M. Grossi, A. Pistoia, On the existence of blowing-up solutions for a mean field equation. Ann. Inst. Henri Poincaré Analyse Non Linéaire 22 (2005), no. 2, 227-257. MR2124164 (2005k:35112)

[10] P. Esposito, M. Musso, A. Pistoia, Concentrating solutions for a planar elliptic problem involving nonlinearities with large exponent, J. Differential Equations 227 (2006), no. 1, 29-68. MR 2233953 (2007f:35085)

[11] C.-S. Lin, C.-L. Wang, Elliptic functions, Green functions and the mean field equation on tori. Ann. of Math. (2) 172 (2010), no. 2, 911-954. MR2680484

[12] C.-S. Lin, S. Yan, Bubbling solutions for relativistic abelian Chern-Simons model on a torus. Comm. Math. Phys. 297 (2010), no. 3, 733-758. MR2653901

[13] M. Nolasco, G. Tarantello, Double vortex condensates in the Chern-Simons-Higgs theory. Calc. Var. Partial Differential Equations 9 (1999), no. 1, 31-91. MR1710938 (2001e:58022)

[14] J. Prajapat, G. Tarantello, On a class of elliptic problems in $\mathbb{R}^{2}$ : Symmetry and uniqueness results. Proc. Roy. Soc. Edinburgh Sect. A 131 (2001), no. 4, 967-985. MR 1855007 (2002j:35108)

[15] G. Tarantello, Multiple condensate solutions for the Chern-Simons-Higgs theory. J. Math. Phys. 37 (1996), no. 8, 3769-3796. MR1400816 (97f:58045)

[16] G. Tarantello, Selfdual gauge field vortices. An analytical approach. Progress in Nonlinear Differential Equations and their Applications, 72. Birkhäuser Boston, Inc., Boston, MA, 2008. MR2403854 (2009k:58028)

[17] A. Tur, V. Yanovsky, Point vortices with a rational necklace: new exact stationary solutions of the two-dimensional Euler equation. Phys. Fluids 16 (2004), no. 8, 2877-2885. MR 2075552 (2005b:76029)

Departamento de Ingeniería Matemática and Centro de Modelamiento Matemático (UMi 2807 CNRS), Universidad de Chile, Casilla 170 Correo 3, Santiago, Chile

E-mail address: delpino@dim.uchile.cl

Dipartimento di Matematica, Università degli Studi "Roma Tre", Largo S. Leonardo Murialdo, 1, 00146 Roma, Italy

E-mail address: esposito@mat.uniroma3.it

Departamento de Matemática, Pontificia Universidad Católica de Chile, Avda, Vicuña Mackenna 4860, Macul, Chile

E-mail address: mmusso@mat.puc.cl 